\title{
Evaluation of the Service Provision of Emergency Obstetric Care in a Tertiary Care Centre: A Clinical Audit
}

\author{
Francis Paul ${ }^{1}$, Kent Kong Kian Keong ${ }^{2 *}$, Jennifer Tan ${ }^{3}$, Anna Lee En Moi ${ }^{3}$, Alen Lim Chung Chieh ${ }^{1}$
}

\author{
${ }^{1}$ Administration Office, \\ Duchess of Kent Hospital, Sandakan, \\ Sabah, Malaysia \\ ${ }^{2}$ Clinical Research Centre, \\ Duchess of Kent Hospital, Sandakan, \\ Sabah, Malaysia \\ ${ }^{3}$ Nursing Department, \\ Duchess of Kent Hospital, Sandakan, \\ Sabah, Malaysia
}

\section{*Corresponding author's email: kentkongkk85@gmail.com}

Keywords: obstetrics emergency, obstetrics emergency protocol, obstetrics red alert

NMRR Research ID:

NMRR-18-2067-42538
Introduction: Maternal death is a sensitive health indicator being monitored closely by the Ministry of Health. Obstetric emergency (OE) protocol is introduced to manage $\mathrm{OE}$ and to improve maternal outcome. However, there is no national OE guideline available and the $\mathrm{OE}$ protocol varies among different institutions. The current audit aims to evaluate the service quality during $\mathrm{OE}$ in Duchess of Kent Hospital (DOKH) in accordance with OE protocol DOKH revision-2017. Methods: This was a retrospective clinical audit on the quality of service provision during $\mathrm{OE}$ from Jan to June 2018 in terms of response time ( $<5$ minutes), presence of discipline team from anaesthesiology and discipline team from obstetrics and gynaecology (O\&G), the appropriateness of triggering obstetrics emergencies and outcome. The audit standard for adherences was set at 95\% (set by DOKH quality assurance team). Results: Total of 29 cases of OE were reported in 2018. Up to 24 $(82.8 \%)$ cases were attended by O\&G team and 17 (58.6\%) cases by anaesthesiology team within a 5-minute response time. Both discipline teams were present on-site during all the OE. A total of 27 (93.1\%) cases were triggered in adherence to the OE protocol. Only $27(93.1 \%)$ cases were attended by specialists from O\&G and 16 (55.2\%) cases by anaesthesiologists respectively. Not all specialists attended the patients on-site but rather depended on their team to report. A total of 12 cases (41\%) were admitted to ICU. The case with mortality was $1(3.45 \%)$ with the 
Borneo Journal of Medical Sciences (BJMS), Special Issue, Volume 3, March 2020: $5-6$

diagnosis of subdural and intraparenchymal bleed after 4 days of ICU care. Conclusion: The overall adherence to OE protocol was not satisfactory. Educational workshops will be conducted among the two disciplines to improve the critical response time to OE. A formal meeting will be conducted to discuss the possible solution to the mandatory review by the specialists for every OE as a strategy to improve the quality of services. 